\title{
Experience with the Levitronix CentriMag circulatory support system as a bridge to decision in patients with refractory acute cardiogenic shock and multisystem organ failure
}

Ranjit John, MD, ${ }^{a}$ Kenneth Liao, MD, ${ }^{a}$ Katherine Lietz, MD, PhD, ${ }^{\mathrm{b}}$ Forum Kamdar, BS, ${ }^{a}$ Monica Colvin-Adams, MD, Andrew Boyle, MD, ${ }^{b}$ Leslie Miller, MD, ${ }^{b}$ and Lyle Joyce, MD, $\mathrm{PhD}^{\mathrm{a}}$

Objective: Patients with refractory acute cardiogenic shock and multisystem organ failure have a poor outcome with implantation of permanent ventricular assist devices. We review our experience with the use of the CentriMag (Levitronix LLC, Waltham, Mass) circulatory support system in such patients whose neurologic status was uncertain.

Methods: From January 2004 to June 2006, 30 patients underwent CentriMag circulatory support system placement at the University of Minnesota. Of these patients, 12 were transferred from an outside hospital with refractory acute cardiogenic shock requiring biventricular support; they are the focus of this study.

Results: Of our 12 study patients, 8 underwent successful bridging to the HeartMate XVE (Thoratec Corp, Pleasanton, Calif) ventricular assist device after biventricular support (mean support time of 9.4 days, range: 5-22 days). Another 2 patients underwent successful explantation (after 8 and 9 days); the remaining 2 patients died (after 4 days). Thus, the survival on CentriMag support, to either bridge or recovery, was $83 \%$ (10/12). Of the 8 patients who subsequently underwent HeartMate implantation, 5 also underwent a heart transplant within 6.9 months (range, 4.5-10 months), another 2 are still awaiting a transplant, and 1 died of sepsis and right ventricular failure 3 days after HeartMate implantation. Thus, for our 12 study patients, long-term survival was $75 \%$ at 1 month and $62.5 \%$ at 1 year.

From the Division of Cardiothoracic Surgery, Department of Surgery ${ }^{\mathrm{a}}$ and Division of Cardiology, ${ }^{\mathrm{b}}$ University of Minnesota, Minneapolis, Minn.

Monica Colvin-Adams reports lecture fees from Medtronic; Andrew Boyle reports consulting and lecture fees from Thoratec; Leslie Miller reports consulting fees and grant support from Thoratec.

Received for publication Sept 26, 2006; revisions received Jan 22, 2007; accepted for publication Jan 29, 2007.

Address for reprints: Ranjit John, MD, Assistant Professor, Division of Cardiothoracic Surgery, 420 Delaware St SE, MMC 207, University of Minnesota, Minneapolis, MN 55455 (E-mail: johnx008@umn.edu).

J Thorac Cardiovasc Surg 2007;134:351-8

$0022-5223 / \$ 32.00$

Copyright (C) 2007 by The American Association for Thoracic Surgery

doi:10.1016/j.jtcvs.2007.01.085
Conclusions: Our aggressive strategy in this group of patients involved early operative intervention and implantation of biventricular support. By using this strategy, we avoided the urgent placement of expensive long-term ventricular assist devices in hemodynamically unstable patients with multisystem organ failure whose neurologic status was uncertain until end-organ recovery and excellent hemodynamic stability were achieved with the relatively inexpensive short-term CentriMag circulatory support system. The excellent midterm outcomes in this group of patients whose original prognosis was poor justify this therapeutic strategy.

$\mathrm{R}$ efractory acute cardiogenic shock remains the leading cause of death in patients hospitalized with myocardial infarction. Multicenter trials have reported an early mortality rate of more than $50 \%$ in such patients despite adequate and prompt revascularization. ${ }^{1,2}$ For many of these patients, mechanical circulatory support as a bridge to transplantation remains the only means of survival. Tremendous progress has been made in the management of acute cardiogenic shock with the use of mechanical assist devices. ${ }^{3-5}$ The increased applicability and excellent results with left ventricular assist devices (LVADs) have revolutionized the treatment options available for patients with end-stage heart failure. ${ }^{6-8}$ The success 


\author{
Abbreviations and Acronyms \\ ACT = activated clotting time \\ $\mathrm{ECMO}=$ extracorporeal membrane oxygenation \\ GCS = Glasgow Coma Scale \\ IABP $=$ intraaortic balloon pump \\ $\mathrm{LVAD}=$ left ventricular assist device \\ MSOF $=$ multisystem organ failure \\ $\mathrm{RVAD}=$ right ventricular assist device \\ $\mathrm{SCr}=$ serum creatinine \\ $\mathrm{VAD}=$ ventricular assist device
}

of the Randomized Evaluation of Mechanical Assistance for the Treatment of Congestive Heart Failure trial of destination therapy further opened a new door for patients ineligible for a heart transplant. ${ }^{9}$

Patients whose survival is still poor are those in multisystem organ failure (MSOF) secondary to acute cardiogenic shock refractory to maximal medical therapy (including the use of intraaortic balloon pump [IABP], multiple inotropes, and pressors). The only 2 options available for such patients are (1) ventricular support with mechanical assist devices or (2) extracorporeal membrane oxygenation (ECMO). For patients with MSOF who have had a prior cardiac arrest or who have severe hemodynamic instability, outcomes after permanent LVAD implantation are extremely poor. The use of LVADs for these patients is controversial. First, these patients often need biventricular support, the presence of which limits long-term options. Second, the question arises as to whether these patients should have a permanent device placed at the time of presentation. The common combination of MSOF and uncertain neurologic status, along with the uncertainty of adequate social support (because of the unavailability of adequate time to complete such an evaluation), clouds the transplant candidacy of these patients. In addition, the major and lengthy surgery required for permanent LVAD implantation in patients with significant hemodynamic instability, coagulopathy, and liver and renal failure significantly increases postoperative morbidity and mortality.

Clearly, there is an increased role for temporary circulatory support in such patients. Questions remain as to the ideal device, the optimal duration of support, and the ideal timing to bridge these patients to a permanent device. A disadvantage with temporary support is that multiple surgeries and the need for anticoagulation are required, both of which have their attendant complications. An advantage, at least in patients in whom MSOF does not resolve and whose neurologic function recovers poorly, is that permanent LVAD implantation is avoided.

We developed an algorithm at the University of Minnesota for initiating temporary circulatory support in patients with refractory acute cardiogenic shock and MSOF who have contraindications to permanent ventricular assist device (VAD) implantation at the time of presentation. We performed a retrospective study of all patients from January 2004 to June 2006 who were transferred to our institution from outside institutions with acute cardiogenic shock refractory to maximal medical therapy and who then underwent temporary CentriMag (Levitronix LLC, Waltham, Mass) circulatory support at our institution. The objectives of our study were to (1) describe the benefit of CentriMag support in these patients, (2) describe postoperative endorgan recovery and neurologic recovery after temporary CentriMag support, (3) identify the timing of permanent device implantation, and (4) describe overall outcomes, including postoperative complications and survival to permanent LVAD implantation, heart transplantation, or device explant.

\section{Materials and Methods Patients}

From January 2004 to June 2006, 30 patients underwent temporary CentriMag circulatory support at the University of Minnesota. Of these 30 patients, 12 were transferred from outside hospitals with refractory acute cardiogenic shock, despite IABP, multiple inotropes and pressors, and MSOF. Of the 12 patients, 2 were transferred to our institution on Bio-Medicus pumps (Medtronic Bio-Medicus Inc, Minneapolis, Minn) because they could not be weaned off cardiopulmonary bypass after emergency coronary artery bypass surgery for acute myocardial infarction. Both of these patients had an episode of cardiac arrest in the catheterization laboratory at the outside institution and were urgently taken for bypass surgery. All 12 patients were supported with the biventricular CentriMag system and thus comprise our study group. The remaining 18 patients underwent CentriMag placement for other indications, including posttransplant allograft failure, right ventricular failure after permanent LVAD, and postcardiotomy shock.

\section{CentriMag System}

The CentriMag system is composed of a single-use centrifugal blood pump, a motor, a console, and a flow probe. ${ }^{10,11}$ The system is based on the "bearingless motor" technology, which combines the drive, magnetic bearing, and rotor function into a single unit. The motor generates the magnetic bearing force that levitates the impeller in the pump housing while also generating the torque necessary to produce unidirectional flow. The characteristic of the magnetic levitation is the absence of bearings and seals, resulting in minimal friction and minimal heat generation in the blood path. The rotor surface is uniformly washed, which minimizes the area of blood stagnation and turbulence in the pump. To reduce shear forces and hemolysis, the mechanical gaps in the pump are greater than $0.6 \mathrm{~mm}$. This system can produce flows up to 10 liters per minute under normal physiologic conditions and has a priming volume of $31 \mathrm{~mL}$.

\section{Surgical Techniques}

All 12 study patients underwent urgent cardiac surgery at our institution through a median sternotomy for initiation of biven- 
tricular CentriMag support. After anticoagulation based on activated clotting time (ACT)-guided heparinization, the patients underwent cardiopulmonary bypass. Aminocaproic acid (Amicar, Amgen Inc, Thousand Oaks, Calif) was administered to all patients. If time permitted, the cannulas used for aortic and right atrial cannulation for cardiopulmonary bypass were tunneled through subcostal incisions (because these same cannulas would be used later for the LVAD outflow and the right ventricular assist device [RVAD] inflow). The urgent need for cardiopulmonary bypass was based on these patients' hemodynamic instability. Further, decompression of the heart with cardiopulmonary bypass facilitated the ease of subsequent placement of the left atrial and pulmonary artery cannulas (also placed after subcostal tunneling).

The aortic and pulmonary artery cannulas were placed through double-layer purse-string 3-0 Tevdek sutures with multiple pledgets. Both atrial cannulas were placed through double-layer purse-string 2-0 Tevdek sutures with multiple pledgets. For additional stability, multiple 2-0 Tevdek ties were placed around the cannula and the snares. The cannula of choice for aortic and pulmonary artery cannulation was the Medtronic elongated 1-piece arterial cannula $(20 \mathrm{~F}$ or $22 \mathrm{~F})$. The choice of cannula for atrial cannulation was an angled venous cannula (24F or $28 \mathrm{~F})$. Note that the choice of cannula size was customized to the patient's body surface area.

The CentriMag pump heads and tubing were prepared and primed extracorporeally. The tubing was then divided within the operating field and appropriately connected to each cannula, carefully avoiding any air in the system. LVAD support was then initiated, followed by RVAD support. The speed of each pump was increased to provide a cardiac index of greater than 2.2 to 2.4 $\mathrm{L} / \mathrm{min}$. Once satisfactory flows were achieved, protamine was given and meticulous hemostasis was performed. The sternotomy was closed in the usual fashion with surgical steel wires, and the patient was returned to the intensive care unit. In case of coagulopathy with ongoing mediastinal bleeding, the patient's chest was packed and left open.

\section{Anticoagulation}

Heparin was only started once the chest tube output was less than $50 \mathrm{~mL}$ per hour (usually $\leq 24$ hours) to maintain an ACT of 160 to 180 seconds for the first 4 days. After day 4, heparin dosing was increased to maintain an ACT of 180 to 200 seconds.

\section{Postoperative Care}

End-organ function was closely monitored. Appropriate enteral or parenteral nutrition was also initiated. Once patients had stable hemodynamics on CentriMag support with improving end-organ function, they were weaned off sedation and allowed to awaken to assess their neurologic function. If the neurologic function remained uncertain, a neurologic consultation was obtained along with appropriate tests (computed tomography scan, electroencephalogram). If neurologic function and prognosis were poor, the family was consulted and a decision was made regarding withdrawal of support (Figure 1).

Given evidence of neurologic recovery, a decision was made to (1) continue with CentriMag support, (2) wean the patient off CentriMag support, (3) evaluate the patient for transplantation, and
(4) bridge the patient to permanent LVAD implantation. The timing for the decision also depended on renal, hepatic, and respiratory recovery and on confirmation of the absence of any infection. Within 3 to 5 days after CentriMag support began, echocardiography was performed to evaluate left and right ventricular recovery. This echocardiography assessment was made during a period of gradual reduction in CentriMag flow and adequate heparinization. If left and right ventricular recovery was present, then explantation was performed once renal, hepatic, and respiratory function returned to normal (Figure 1).

If evidence of ventricular recovery was not seen within 1 week after CentriMag support began, a decision was made on eligibility for implantation of a HeartMate XVE LVAD (Thoratec Corp, Pleasanton, Calif) as either bridge or destination therapy. During HeartMate implantation, the existing aortic and right atrial cannulas were used for cardiopulmonary bypass after CentriMag support was discontinued. Aprotinin was used during HeartMate implantation. A preperitoneal $(n=5)$ or an intraperitoneal $(n=3)$ HeartMate implantation was performed. After weaning from cardiopulmonary bypass, LVAD flow was initiated and the patients were supported with appropriate inotropes or pressors as necessary.

\section{Statistical Analysis}

Differences in hemodynamic and end-organ function were compared using 1-way repeated analysis of variance. Survival estimates were based on the Kaplan-Meier method and compared using log-rank statistics. All values are expressed as mean \pm standard error of the mean. All data were analyzed using the SAS System software version 9.0 (SAS Institute, Inc, Cary, NC).

\section{Results \\ Patient Characteristics}

In our study, the 12 patients who underwent temporary biventricular CentriMag support were all male; their mean age was $57.9 \pm 9$ years. The cause of cardiogenic shock was acute myocardial infarction $(\mathrm{n}=10)$ or acute decompensation of chronic ischemic cardiomyopathy $(n=2)$. The average duration of CentriMag support was 8 days (range 4-22 days).

The patients' hemodynamic characteristics and end-organ functional status before and on the last day of CentriMag support are summarized in Table 1 . All 12 patients initially had evidence of severe biventricular failure and required mechanical ventilation and intravenous infusion of inotropes and vasopressors. Eleven patients had IABPs $(91.7 \%)$.

At the time CentriMag support began, 9 patients $(75 \%)$ were unresponsive, with a Glasgow Coma Scale (GCS) score of $3 ; 2$ patients had significantly impaired responsiveness, with a GCS score of 7; and 1 patient had a GCS score of 11. The mean arterial pressure was $67.0 \pm 9.8 \mathrm{~mm} \mathrm{Hg}$, and the mean central venous pressure was $17.1 \pm 3.9 \mathrm{~cm}$ $\mathrm{H}_{2} \mathrm{O}$. Eleven patients $(91.7 \%)$ had evidence of significant renal dysfunction with a serum creatinine $(\mathrm{SCr})$ concentration 


\section{Acute Refractory Cardiogenic Shock \\ (Multiple Inotropes \& Pressors, IABP) \\ with \\ Multisystem Organ Failure +/- Prior Cardiac Arrest \\ Uncertain Neurologic Status \\ Transfer to our institution \\ CentriMag Biventricular Support}

(Monitor End-organ Function, Nutritional Support, Wean Sedation, Echocardiography)
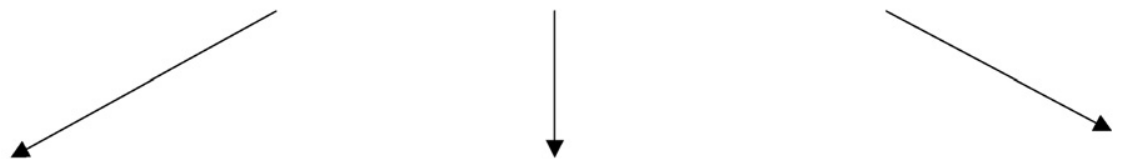

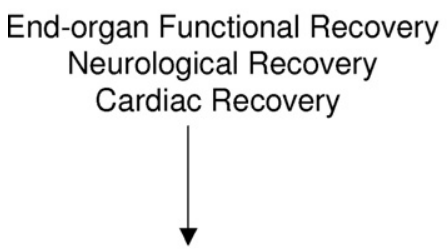

CentriMag Explant
End-organ Functional Recovery Neurological Recovery No Cardiac Recovery

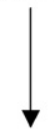

HeartMate Implantation
End-organ Functional Recovery +/No Neurological Recovery Cardiac Recovery +/-

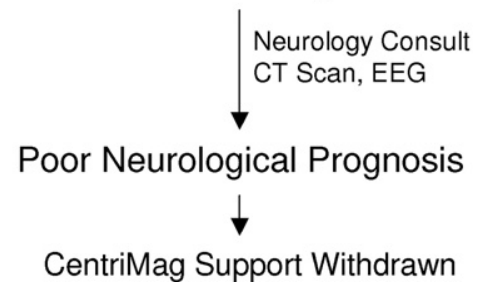

Figure 1. Algorithm depicting the management of patients transferred from outside institutions with refractory acute cardiogenic shock with MSOF. IABP, Intraaortic balloon pump; CT, computed tomography; EEG, electroencephalogram.

of $2 \mathrm{mg} / \mathrm{dL}$ or greater (mean, $2.67 \pm 0.9 \mathrm{mg} / \mathrm{dL}$ ) and a mean estimated glomerular filtration rate of $44.5 \pm 26 \mathrm{~mL} / \mathrm{min} /$ $\left.1.73 \mathrm{~m}^{2}\right)$. All patients had abnormalities in liver function. Ten patients $(83.3 \%)$ had evidence of systemic inflammation with leukocytosis greater than $10 \times 10^{3} / \mu \mathrm{L}$; for all 12 patients, the mean white blood cell count was $15.2 \pm 7.4 \times$ $10^{3} / \mu \mathrm{L}$.

\section{Short-term Survival}

Of the 12 patients, 8 underwent successful bridging to HeartMate implantation after CentriMag support (mean support time 9.4 days; range 5-22 days); 2 patients underwent successful explantation (after 8 and 9 days); and the remaining 2 patients died (after 4 days of support). The 2 patients who died both had CentriMag support withdrawn because of severe neurologic injury (possibly preexisting). Thus, the survival on CentriMag support, to either HeartMate implantation or recovery, was $83 \%(10 / 12)$, with an overall mean circulatory support time of 8 days (range 4-22 days). The probability of survival on CentriMag support was $83 \% 7$ days after implantation and $83 \% 14$ days after implantation.

\section{Long-term Survival}

Of the 8 patients who subsequently underwent HeartMate implantation, 5 also underwent a heart transplant within 6.9 months (range 4.5-10 months); another 2 patients are still awaiting a transplant at 51 and 77 days after implantation, and 1 patient died of sepsis and right ventricular failure 3 days after implantation. Of the 5 heart transplant recipients, 4 are still alive at 18.6 months posttransplant (range 15-23 months) and 1 died of sepsis 3 days posttransplant surgery. Thus, the survival after HeartMate implantation to a heart transplant was $62 \%(5 / 8)$. The 2 patients who underwent successful explantation (after 8 and 9 days of CentriMag support) were free of heart failure at 18.6 months follow-up.

Thus, the total long-term survival benefit of CentriMag support (as either "bridge-to-bridge" or "bridge-to-recovery" therapy) in our 12 patients was $75 \%$ at 1 month and $62.5 \%$ at 1 year, as shown in Figure 2.

\section{Post-CentriMag Support Measurements}

To assess changes in hemodynamic, neurologic, and endorgan function, we compared hemodynamic parameters, 
TABLE 1. Hemodynamic characteristics and end-organ functional status before and at the end of CentriMag (Levitronix LLC, Waltham, Mass) support

Before CentriMag support

( $N=12)$

Hemodynamic characteristics (mean)

Systolic blood pressure $(\mathrm{mm} \mathrm{Hg})$

Diastolic blood pressure $(\mathrm{mm} \mathrm{Hg})$

Arterial pressure $(\mathrm{mm} \mathrm{Hg})$

Heart rate (beats/min)

Systolic pulmonary artery pressure $(\mathrm{mm} \mathrm{Hg}$ )

Diastolic pulmonary artery pressure $(\mathrm{mm} \mathrm{Hg})$

Mean pulmonary artery pressure $(\mathrm{mm} \mathrm{Hg})$

Central venous pressure $\left(\mathrm{cmH}_{2} \mathrm{O}\right)$

Laboratory parameters (mean) $\mathrm{pH}$

White blood count (cells $\times 10^{3} / \mu \mathrm{L}$ )

Hemoglobin (mg/dL)

Hematocrit (\%)

Platelet count (cells $\times 10^{3} / \mu \mathrm{L}$ )

Blood urea nitrogen $(\mathrm{mg} / \mathrm{dL})$

$\mathrm{SCr} \geq 2 \mathrm{mg} / \mathrm{dL}$

$\mathrm{SCr}(\mathrm{mg} / \mathrm{dL})$

Estimated $\mathrm{SCr}$ clearance $\left(\mathrm{mL} / \mathrm{min} / 1.73 \mathrm{~m}^{2}\right)$

AST

ALT

ALP

Total bilirubin

Albumin

INR

PTT
On last day of CentriMag support

( $N=12)$

$104.1 \pm 19.5$

$66.1 \pm 18.4$

$78.7 \pm 13.7$

$82.7 \pm 14.4$

$33.0 \pm 8.1$

$18.7 \pm 4.0$

$48.1 \pm 9.2$

$13.7 \pm 2.7$

$7.36 \pm 0.07$

$13.2 \pm 6.8$

$10.1 \pm 1.0$

$29.8 \pm 3.4$

$116.9 \pm 69.9$

$49.4 \pm 31.4$

$5(41.7 \%)$

$2.27 \pm 1.3$

$56.5 \pm 39.5$

$251 \pm 292$

$87 \pm 94$

$103.7 \pm 67.9$

$2.85 \pm 2.8$

$2.7 \pm 0.3$

$1.9 \pm 1.2$

$59.7 \pm 31.9$
One-way ANOVA $P$ value

.06

.16

.03

.29

.07

.03

.43

.05

.48

.99

.20

.33

.52

.47

.32

.12

.23

.05

.04

.008

.38

.90

.31

ANOVA, Analysis of variance; $S C r$, serum creatinine; $A S T$, aspartate aminotransferase; $A L T$, alanine aminotransferase; $A L P$, alkaline phosphatase; INR, international normalized ratio; $P T T$, prothrombin time.

laboratory markers of end-organ function, and neurologic function using the GCS before and on the last day of CentriMag support. The last day was the day of permanent HeartMate implantation, or CentriMag explantation, or day of death.

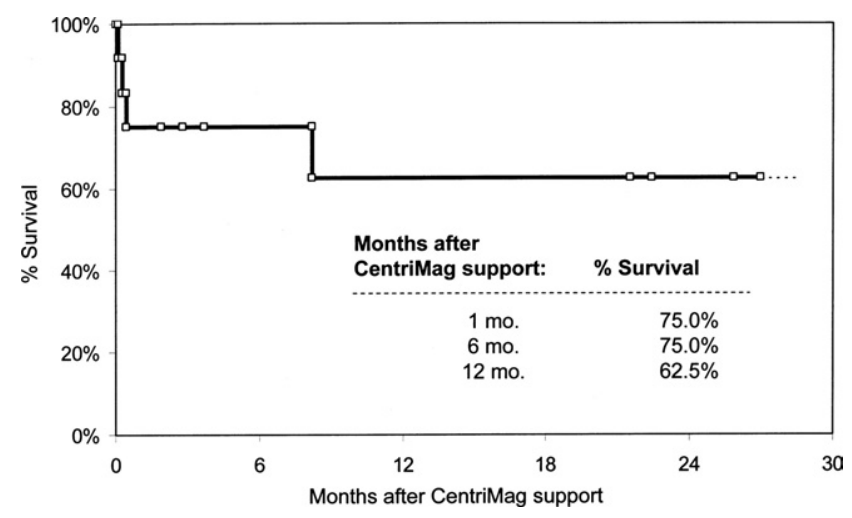

Figure 2. Kaplan-Meier survival after CentriMag (Levitronix LLC, Waltham, Mass) support.
Hemodynamic changes. Most measures of left ventricular function and right heart function improved on CentriMag support (Table 1). Six patients (50\%) were able to be completely weaned off inotropes while on CentriMag support.

Neurologic recovery. Patients on CentriMag support had marked improvement in neurologic status. On the last day of CentriMag support, neurologic function had significantly improved in 7 of 9 patients who initially were unresponsive with a GCS score of 3; of those 7 patients, 4 completely recovered. In 2 patients with moderately decreased responsiveness and an initial GCS score of 7, neurologic function returned to near-normal with a GCS score greater than 11. The GCS score remained unchanged in the 1 patient with initially intact neurologic function.

End-organ function. Significant changes in renal and hepatic function occurred on CentriMag support (Table 1). Of the 11 patients whose initial SCr was greater than $2 \mathrm{mg} / \mathrm{dL}, 5 \mathrm{had}$ improvement in renal function, with a decline in $\mathrm{SCr}$ of less than $2 \mathrm{mg} / \mathrm{dL}$ (45\%). Markers of acute hepatic injury, such as elevations in liver enzymes, significantly decreased. 
CentriMag performance. Adequate circulatory support was achieved in all 12 patients to maintain a cardiac index greater than 2.2 liters per minute. Both left and right CentriMag pumps were run between 2500 and $3300 \mathrm{rpm}$ to provide a flow of 4 to 6 liters per minute. Arterial pulse was usually absent for the first 48 hours. With LV recovery, pulsatility returned, suggestive of native LV ejection. No pump failure occurred because of thrombosis or any other mechanical causes. One patient who received heparin for cardiopulmonary bypass had evidence of extensive thrombus in the LVAD outflow tubing within 30 minutes after CentriMag support began; this patient was diagnosed with heparin-induced thrombocytopenia. After administration of bivalirudin, the tubing and CentriMag pump were exchanged and functioned well subsequently.

\section{Discussion}

In most of our 12 patients with acute cardiogenic shock, with MSOF and severe neurologic dysfunction, we showed that temporary biventricular CentriMag circulatory support improved end-organ function and allowed for full neurologic recovery; $80 \%$ of them became acceptable candidates for HeartMate implantation. Our approach allowed for recovery of many patients otherwise ineligible for HeartMate LVAD implantation, with an excellent 1-year survival.

As the use of mechanical circulatory support in patients with heart failure increases, and as options increase for mechanical assistance, the indications for implantation of each device needs to be clarified. Although individual patients continue to benefit from this life-saving technology, the cost-benefit ratio of implantable support therapy certainly does not justify its use in all potential recipients. Identification of preoperative variables that accurately predict risk is important for planning and devising management strategies, especially in patients with medical challenges. A Columbia-Presbyterian Hospital study identified the following risk factors for increased operative mortality: mechanical ventilation, postcardiotomy shock, previous LVAD, elevated central venous pressure, and elevated prothrombin time. ${ }^{12,13}$ The presence of those factors (score $>5$ ) was associated with an almost $50 \%$ postoperative mortality rate. Note that their screening scale did not even take into account the patient's neurologic status. In addition to the tremendous impact on financial resources and logistics, the emotional impact on the family when support is withdrawn from a patient who is brain-dead or has severe neurologic injury is devastating.

We do not agree with the concept that a long-term or permanent device should always be placed in an urgent situation. ${ }^{14,15}$ In our experience, rapid stabilization of the patient was of primary importance: Adequate systemic perfusion must be provided with minimal complications, neurologic function must be ascertained, and end-organ func- tion must be allowed to recover. Screening scales suggest that permanent LVAD implantation in patients with MSOF has an unacceptably high mortality rate. ${ }^{13}$ Even though hemodynamic stability is fairly comparable after short-term and permanent LVAD implantation, there will be a cohort of patients who have irreversible neurologic injury and in whom placement of a permanent LVAD is simply a waste of resources, with no gain achieved.

Two important questions must be considered in patients with acute cardiogenic shock, MSOF, a prior cardiac arrest, and severe neurologic dysfunction. First, how do we identify the patient who will benefit from temporary circulatory support? Mortality rates after permanent LVAD insertion in this group are greater than $50 \%$. Second, what modality of temporary circulatory support should be used? This is a relatively unresolved issue. Short-term VADs include the widely used Abiomed BVS 5000 (Abiomed Inc, Danvers, Mass) and Bio-Medicus systems and the more recently introduced CentriMag system. ${ }^{10,16-18}$ In addition, ECMO is also useful. ${ }^{19-21}$ Samuels and colleagues ${ }^{16}$ reported a $31 \%$ hospital discharge rate for patients with acute cardiac failure on the Abiomed. The many advantages of the Abiomed have led to an extensive experience with it. However, disadvantages include the need for performing anastomoses for aortic and pulmonary artery cannulation, with resultant bleeding complications and the need for pump exchanges at less than 1-week intervals.

Although ECMO has multiple advantages, major disadvantages include a limited duration of support, a high incidence of complications with increasing duration of support, and the need for fairly stringent anticoagulation. In addition, ECMO requires a dedicated team of personnel to allow for its safe use. Pagani and colleagues, ${ }^{19}$ of the University of Michigan, reported a 43\% 1-year survival in patients with cardiogenic shock with either a prior cardiac arrest or severe hemodynamic instability who were initially treated with ECMO as a bridge to permanent LVAD implantation. In contrast, a Columbia-Presbyterian Hospital study reported a 30 -day survival of $56 \%$ in 46 patients who underwent urgent HeartMate LVAD implantation after being transferred from outside institutions with cardiogenic shock. ${ }^{22}$

In our study, by using CentriMag support in 12 patients with refractory acute cardiogenic shock and MSOF who were transferred from an outside hospital, we achieved a 30 -day survival of $75 \%$. The ease of use of the CentriMag system allowed for rapid assessment of ventricular recovery, weaning, and potential explantation (as we were able to achieve in 2 patients). Its "user-friendly" nature is borne out by the fact that virtually any cannulas (including those used for routine cardiopulmonary bypass) can be used. In addition, 2 patients who were transferred with Bio-Medicus centrifugal pumps were easily converted to extracorporeal CentriMag support. The CentriMag system's durability is 
also much greater than that of any other temporary circulatory assist device currently available.

In a study from Innsbruck, Hoefer and colleagues ${ }^{23}$ reported excellent results with ECMO support in patients with cardiogenic shock. However, they reported a 50\% mortality rate in patients with ECMO support who were bridged to permanent VAD implantation; most deaths due to MSOF with sepsis. The limited durability of ECMO (the risk of complications increases with increased duration of ECMO) may have led to the institution of permanent VAD support while patients still had unresolved end-organ dysfunction.

Our policy at the University of Minnesota for such patients allows for recovery of renal, hepatic, and respiratory systems during the support period. These patients can also be weaned off the ventilator and even extubated if their condition warrants. While the patient is intubated, nursing and physiotherapy staff are encouraged to mobilize the patient and perform some rehabilitative therapy. We have also instituted an aggressive nutritional program (preferably enteral) during CentriMag support. Although we have not mobilized patients out of bed, they are awake and can follow commands, which allows us to also perform limited physical therapy.

Additional advantages with CentriMag support include the absence of stringent requirements for anticoagulation. Many patients who require urgent mechanical circulatory support are receiving clopidogrel or glycoprotein IIb/IIIa inhibitors; as a result, they have significant postoperative bleeding that is often refractory to correction of coagulopathy. In such patients, we have often withheld anticoagulation with heparin for 48 to 72 hours, until all mediastinal bleeding resolves. Even so, in our current study, we did not have any pump malfunction due to thrombosis or thromboembolic events. When anticoagulation was withheld, flows greater than 4 liters per minute reduced the risk of thrombosis even in the absence of anticoagulation.

Experience with ECMO has revealed fairly stringent requirements for anticoagulation and a high incidence of thrombotic complications when heparin is not used. Because a majority of these patients on CentriMag support are already on IABP support, we continue IABP support in addition to biventricular assist device support for 24 to 72 hours to allow for some degree of pulsatile perfusion. Some reports suggest beneficial effects of afterload reduction on myocardial recovery and improved end-organ function with pulsatile flow. ${ }^{24}$ In our study, we showed that despite the need for multiple surgeries (or at least a second operation for explantation of the temporary VAD system and for permanent LVAD implantation), satisfactory results can be achieved by using CentriMag support as a bridge to explantation, recovery, or permanent LVAD implantation in patients with refractory cardiogenic shock and MSOF.
The recent introduction of percutaneous VADs into the clinical armamentarium has provided yet another option for patients with cardiogenic shock in select situations. Although gaining popularity as temporary support during high-risk percutaneous coronary interventions, the TandemHeart (CardiacAssist Inc, Pittsburgh, Pa) percutaneous VAD has also been successfully used for patients in cardiogenic shock. Although the primary advantage is the relative rapidity of institution of hemodynamic support, potential disadvantages include the relative limitation of flow (maximum up to 4 liters per minute), thereby restricting its use in larger patients. Other disadvantages include the lack of percutaneous support options for the right ventricle, possibility of cannula dislodgment, lower extremity ischemia, and the need for strict anticoagulation. ${ }^{25,26}$

We believe that a team approach is essential for optimizing outcomes even before the patient is transferred to the referral hospital. Honest communication with the primary physicians regarding the options available will help the family realize that even with referral to a major tertiary center the patient may not survive. As soon as the patient and the family arrive, it is essential to clearly depict the long and arduous road ahead with all possible scenarios. Understandably, though, the families are almost always in a state of shock, so exceptional understanding, patience, and time are necessary in dealing with them.

The limitations of our study are those associated with a retrospective study involving a relatively small cohort of patients. Nevertheless, we believe our conclusions are valid: Our 12 patients had a similar clinical profile and were cared for using a single algorithm (Figure 1) based on temporary CentriMag support as bridge therapy. We found CentriMag support to be safe and cost-effective. It allows for maximal use of limited resources and has excellent short-term survivals.

We thank Mary E. Knatterud, PhD, for editorial assistance.

\section{References}

1. Hochman JS, Sleeper LA, Webb JG, Sanborn TA, White HD, Talley JD, et al. Early revascularization in acute myocardial infarction complicated by cardiogenic shock. N Engl J Med. 1999;341:625-35.

2. Urban P, Stauffer JC, Bleed D, Khatchatrian N, Amann W, Bertel O, et al. A randomized evaluation of early revascularization to treat shock complicating acute myocardial infarction: the (Swiss) multicenter trial of angioplasty for shock-(S)MASH. Eur Heart J. 1999;20:1030-8.

3. Spencer FC, Eiseman B, Trinkle JK, Rodd NP. Assisted circulation for cardiac failure following intracardiac surgery with cardiorespiratory bypass. J Thorac Cardiovasc Surg. 1965;49:56-73.

4. DeBakey ME. Left ventricular bypass pump or cardiac assistance. Am J Cardiol. 1971;27:3-11.

5. Morgan J, John R, Rao V, Weinberg A, Lee BJ, Mazzeo PA, et al. Bridging to transplant with the Heartmate left ventricular assist device: The Columbia-Presbyterian twelve-year experience. J Thorac Cardiovasc Surg. 2004;127:1309-16.

6. Dang N, Topkara V, Leacche M, John R, Oz M, Byrne J, et al. Left ventricular assist device implantation following acute anterior wall myocardial infarction and cardiogenic shock: a two-center study. J Thorac Cardiovasc Surg. 2005;130:693-8. 
7. Frazier OH, Rose EA, McCarthy P, Burton NA, Tector A, Levin H, et al. Improved mortality and rehabilitation of transplant candidates treated with a long-term implantable left ventricular assist system. Ann Surg. 1995;222:327-38.

8. DeRose JJ, Umana JP, Argenziano M, Catanese KA, Levin HR, Sun $\mathrm{BC}$, et al. Improved results for postcardiotomy cardiogenic shock with the use of implantable left ventricular assist devices. Ann Thorac Surg. 1997;64:1757-63.

9. Rose EA, Gelijns AC, Moskowitz AJ, Heitjan DF, Stevenson LW, Dembitsky W, et al. Randomized Evaluation of Mechanical Assistance for the Treatment of Congestive Heart Failure (REMATCH) study group. Long-term mechanical left ventricular assistance for end-stage heart failure. N Engl J Med. 2001;345:1435-43.

10. De Robertis F, Birks EJ, Rogers P, Dreyfus G, Pepper JR, Khagani A. Clinical performance with the Levitronix CentriMag short-term ventricular assist device. J Heart Lung Transplant. 2006;25:181-6.

11. Mueller JP, Kuenzli A, Reuthebuch O, Dasse K, Kent S, Zuend G, et al. The CentriMag: a new optimized centrifugal blood pump with levitating impeller. Heart Surg Forum. 2004;7:E477-80.

12. Oz MC, Goldstein DJ, Pepino P, Weinberg AD, Thompson SM, Catanese KA, et al. Screening scale predicts patients successfully receiving long-term implantable left ventricular assist devices. Circulation. 1995;92(suppl II):169-173.

13. Rao V, Oz MC, Flannery MA, Catanese KA, Argenziano M, Naka Y. Revised screening scale to predict survival after insertion of a left ventricular assist device. J Thorac Cardiovasc Surg. 2003;125: 855-62.

14. Williams M, Casher J, Joshi N, Hankinson T, Warren M, Oz M, et al. Insertion of a left ventricular assist device in patients without thorough transplant evaluations: a worthwhile risk? J Thorac Cardiovasc Surg. 2003;126:436-41.

15. Morris R, Pochettino A, O’Hara M, Gardner T, Acker MA. Emergent mechanical support in the community: improvement with early transplant referral. J Heart Lung Transplant. 2005;24:764-8.

16. Samuels LE, Holmes EC, Thomas MP, Entwistle JC III, Morris RJ, Narula J, et al. Management of acute cardiac failure with mechanical assist: experience with the Abiomed BVS 5000. Ann Thorac Surg. 2001;71:S67-72.

17. Morgan JA, Stewart AS, Brian J, Oz MC, Naka Y. Role of the Abiomed BVS 5000 device for short-term support and bridge to transplantation. ASAIO J. 2004;50:360-3.

18. Couper GS, Dekkers RJ, Adams DH. The logistics and cost-effectiveness of circulatory support: advantages of the Abiomed BVS 5000. Ann Thorac Surg. 1999;68:646-9.

19. Pagani FD, Lynch W, Swaniker F, Dyke DB, Bartlett R, Koelling T, et al. Extracorporeal life support to left ventricular assist device bridge to heart transplant. A strategy to optimize survival and resource utilization. Circulation. 1999;100:II206-10.

20. Pagani FD, Aaronson KD, Swaniker F, Bartlett RH. The use of extracorporeal life support in adult patients with primary cardiac failure as a bridge to implantable left ventricular assist device. Ann Thorac Surg. 2001;71:77-81.

21. Smedira NG, Moazami N, Golding CM, McCarthy PM, AppersonHansen C, Blackstone EH, et al. Clinical experience with 202 adults receiving extracorporeal membrane oxygenation for cardiac failure: survival at 5 years. J Thorac Cardiovas Surg. 2001;122:92-102.

22. Kherani AR, Cheema FH, Oz MC, Fal JM, Morgan JA, Topkara VK, et al. Implantation of a left ventricular assist device and the hub-andspoke system in treating cardiogenic shock: who survives? J Thorac Cardiovasc Surg. 2003;126:1634-5.

23. Hoefer D, Ruttmann E, Poelzl G, Kilo J, Hoermann C, Margreiter R, et al. Outcome evaluation of the bridge to bridge concept in patients with cardiogenic shock. Ann Thorac Surg. 2006;82:28-34.

24. Lazar HL, Treanor P, Xang XM, Rivers S, Bernard S, Shemin RJ. Enhanced recovery of ischemic myocardium by combining percutaneous bypass with intra-aortic balloon pump support. Ann Thorac Surg. 1994;57:663-8.

25. Thiele H, Lauer B, Hambrecht R, Boudriot E, Cohen HA, Schuler G. Reversal of cardiogenic shock by percutaneous left atrial-to-femoral arterial bypass assistance. Circulation. 2001;104:2917-22.

26. Kar B, Adkins LE, Civitello AB, Loyalka P, Palanichamy N, Gemmato CJ, et al. Clinical experience with the TandemHeart percutaneous ventricular assist device. Tex Heart J. 2006;33:111-5. 